\title{
Identidade de modelos não lineares para comparar curvas de crescimento de bovinos da raça Tabapuã
}

\author{
Antonio Policarpo Souza Carneiro(1), Joel Augusto Muniz(2), Paulo Luiz Souza Carneiro(3), \\ Carlos Henrique Mendes Malhado(3), Raimundo Martins Filho( ${ }^{(4)}$ e Fabyano Fonseca e Silva ${ }^{(1)}$
}

\begin{abstract}
(1)Universidade Federal de Viçosa, Departamento de Estatística, Avenida Peter Henry Rolfs, s/no, CEP 36570-000 Viçosa, MG, Brasil. E-mail: policarpo@ufv.br, fabyanofonseca@ufv.br (2)Universidade Federal de Lavras, Departamento de Ciências Exatas, Setor de Estatística e Experimentação, Campus Universitário, Caixa Postal 3.037, CEP 37200-000 Lavras, MG, Brasil. E-mail: joamuniz@dex.ufla.br (3)Universidade Estadual do Sudoeste da Bahia, Departamento de Ciências Biológicas, Avenida José Moreira Sobrinho, s/no, Jequiezinho, CEP 45200-000 Jequié, BA, Brasil. E-mail: plscarneiro@gmail.com, carlosmalhado@gmail.com ${ }^{(4)}$ Universidade Federal do Cariri, Avenida Tenente Raimundo Rocha, s/no, Cidade Universitária, CEP 63000-000 Juazeiro do Norte, CE, Brasil. E-mail: rmartinsfilho@yahoo.com.br
\end{abstract}

Resumo - O objetivo deste trabalho foi avaliar o uso de teste de identidade de modelos não lineares, na comparação de curvas de crescimento de bovinos da raça Tabapuã, de cinco regiões de produção do Nordeste do Brasil. Foram analisados dados de peso de 3.695 machos e 4.236 fêmeas, originários das regiões Maranhão, Gado Algodão, Mata Agreste, Sertão e Itapetinga-Valadares. Após ajuste do modelo Brody, aplicou-se o teste da razão de verossimilhança, com aproximação de qui-quadrado, para avaliar a igualdade de parâmetros de curvas de crescimento entre as regiões. O modelo reduzido, com igualdade da taxa de maturidade, com 14 parâmetros, foi o mais adequado para descrever o crescimento dos animais. As curvas de crescimento dos machos apresentaram taxas de maturidade em comum nas regiões de produção Gado Algodão e Mata Agreste, Maranhão e Itapetinga-Valadares, e Sertão. Para as fêmeas, as regiões com taxas de maturidade em comum foram: Mata Agreste e Sertão, Maranhão e Itapetinga-Valadares, e Gado Algodão. A utilização de uma única curva não é adequada para descrever o crescimento de bovinos da raça Tabapuã nas regiões estudadas.

Termos para indexação: modelo Brody, Nordeste brasileiro, regiões de produção, teste da razão de verossimilhança.

\section{Identity of nonlinear models to compare growth curves of the cattle breed Tabapuã}

\begin{abstract}
The objective of this work was to evaluate the use of identity of nonlinear models to compare growth curves of the cattle breed Tabapuã, between five production regions from Northeast Brazil. Weight data of 3,695 males and 4,236 females from Maranhão, Gado Algodão, Mata Agreste, Sertão, and Itapetinga-Valadares regions were analyzed. After adjusting the Brody model, the likelihood ratio test was applied, with a chi-square approximation, to evaluate the equality of parameters of growth curves between those regions. The reduced model with equal rate of maturity for some regions, with 14 parameters, was the most appropriate for describing animal growth. Curves of male growth showed maturity rates in common in the following regions of production: Gado Algodão and Mata Agreste, Maranhão and Itapetinga-Valadares, and Sertão. For females, regions with maturity rates in common were: Mata Agreste and Sertão, Maranhão and Itapetinga-Valadares, and Gado Algodão. The use of a single curve is not appropriate to describe the growth of the cattle breed Tabapuã in the studied regions.
\end{abstract}

Index terms: Brody model, Brazilian Northeast, production regions, likelihood ratio test.

\section{Introdução}

O crescimento dos animais influencia diretamente com a quantidade e a qualidade da carne produzida. Assim, estudos relacionados a curvas de crescimento têm aplicação estratégica em programas de melhoramento genético, auxiliam na definição de critérios de seleção quanto à precocidade de acabamento e à velocidade de ganho de peso e podem auxiliar na definição de sistemas de produção mais eficientes, para cada raça e região, quanto ao manejo dos animais, a programas alimentares, bem como na definição de cruzamentos (Souza et al., 2010; Silva et al., 2011a).

As medidas de peso dos animais distribuem-se ao longo do tempo de forma semelhante a curvas sigmoides e podem ser descritas por modelos que consideram a relação não linear entre peso e idade. 
Assim, para o estudo do crescimento biológico de diferentes espécies, os modelos não lineares são mais adequados para explicar o processo de crescimento (Ratkowsky, 1990).

Os modelos não lineares, geralmente, fornecem bom ajuste, com menos parâmetros do que os modelos lineares, e apresentam parâmetros ou funções destes com interpretação biológica, o que facilita o estudo do peso do animal adulto, da velocidade de crescimento e de pontos críticos de mudanças na velocidade e na taxa de crescimento (Souza et al., 2010; Silva et al., 2011a).

A descrição de curvas de crescimento de bovinos de raças zebuínas, que utiliza modelos não lineares, foi apresentada em vários estudos (Oliveira et al., 2000; Garnero et al., 2005; Santoro et al., 2005; Forni et al., 2007; Malhado et al., 2009; Souza et al., 2010; Silva et al., 2011a, 2011b), nos quais se realizaram ajustes de modelos que descrevem a curva de crescimento dos animais para uma região ou estado. Quando se considera a grande diversidade de ambientes de produção no Brasil, o ajuste de uma única curva de crescimento para diferentes ambientes pode não ser adequado.

Arruda \& Sugai (1994) realizaram a regionalização da pecuária bovina no Brasil, tendo identificado e caracterizado 44 regiões de produção, com características homogêneas quanto ao sistema de produção e ao ambiente geral de criação. No Nordeste, foram identificadas 11 regiões de produção, em que duas abrangem parte de estados vizinhos como Goiás, Minas Gerais e Espírito Santo.

A região Nordeste apresenta grande variabilidade quanto ao sistema de produção, e a maioria de seus estados apresenta duas ou mais regiões de produção bastante heterogêneas. Pernambuco, por exemplo, tem áreas pertencentes a três regiões de produção (Gado Algodão, Mata Agreste e Sertão). Assim, o ajuste de curvas de crescimento específicas, para cada região de produção ou para grupos de regiões similares, pode permitir a identificação de padrões de crescimento mais próximos da realidade da pecuária bovina da região, além de tornar mais eficiente a definição de estratégias de melhoramento genético, nutrição e manejo para bovinos.

Porém, após o ajuste de uma curva de crescimento específica para cada região de produção, é necessário avaliar se o conjunto de equações ajustadas apresenta parâmetros comuns. Para verificar a igualdade de parâmetros e a identidade de modelos de regressão não linear, Regazzi \& Silva (2010) relataram detalhadamente a aplicação do teste da razão de verossimilhança, com aproximação pela estatística qui-quadrado. A identidade de modelos vem sendo aplicada em estudos de desenvolvimento de plantas e de animais, com o objetivo de verificar a possibilidade de ajuste de equações comuns para diferentes grupos de indivíduos (Sarmento et al., 2006; Martins et al., 2011; Santos et al., 2012).

O objetivo deste trabalho foi avaliar o uso do teste de identidade de modelos não lineares, na comparação de curvas de crescimento de bovinos da raça Tabapuã de cinco regiões de produção do Nordeste do Brasil.

\section{Material e Métodos}

Para o experimento, utilizaram-se dados de animais da raça Tabapuã criados no Nordeste brasileiro e provenientes do controle de desenvolvimento ponderal da Associação Brasileira dos Criadores de Zebu (Tabela 1). Tais dados, coletados entre 1970 e 2006, referem-se ao peso corporal do nascimento até no mínimo dois anos de idade de 4.236 fêmeas e 3.695 machos, criados nas cinco principais regiões de produção do Nordeste brasileiro: Maranhão (MA), Gado Algodão (GA), Mata Agreste (AG), Sertão (SE) e Itapetinga-Valadares (IT).

Para o ajuste das curvas de crescimento, consideraram-se apenas animais com pelo menos seis pesagens, o que reduziu o conjunto de dados original

Tabela 1. Descrição dos dados de pesagens: número de animais $(\mathrm{N})$, número médio de pesagens por animal (NMP), pesos mínimo e máximo (Pmin - Pmax, kg) e idade máxima (IM, dias), para fêmeas e machos da raça Tabapuã de cinco regiões de produção do Nordeste.

\begin{tabular}{lcccc}
\hline Regiões $^{(1)}$ & N & NMP & Pmin-Pmax & IM \\
\hline MA - fêmeas & 191 & 6,5 & $31-592$ & 866 \\
MA - machos & 29 & 6,4 & $38-611$ & 734 \\
GA - fêmeas & 88 & 6,7 & $32-575$ & 854 \\
GA - machos & 105 & 6,7 & $35-607$ & 876 \\
AG - fêmeas & 536 & 6,8 & $32-595$ & 873 \\
AG - machos & 415 & 6,7 & $33-615$ & 989 \\
SE - fêmeas & 39 & 7,2 & $31-416$ & 760 \\
SE - machos & 31 & 6,7 & $38-505$ & 728 \\
IT - fêmeas & 3.382 & 6,6 & $29-595$ & 1090 \\
IT - machos & 3.115 & 6,6 & $36-604$ & 1056 \\
\hline
\end{tabular}

(1)MA, Maranhão; GA, Gado Algodão; AG, Mata Agreste; SE, Sertão; IT, Itapetinga-Valadares. 
de 25.920 para 7.931 animais (Tabela 1). Informações das demais regiões de produção do Nordeste não foram incluídas nas análises, em razão do reduzido número de animais com pelo menos seis pesagens.

Para a descrição da curva de crescimento dos animais, ajustou-se o modelo Brody aos dados de peso e idade, conforme

$$
\mathrm{y}_{\mathrm{i}}=\mathrm{A}\left(1-\mathrm{be}^{-\mathrm{kx}}\right)+\varepsilon_{\mathrm{i}}
$$

em que: $\mathrm{y}_{\mathrm{i}}$ é o peso do animal i na idade $\mathrm{x}_{\mathrm{i}}$; A representa o valor assintótico, interpretado como peso do animal adulto ou peso à maturidade; b é parâmetro de escala, que é uma constante de integração, geralmente sem interpretação biológica; $\mathrm{k}$ é o parâmetro mais importante, pois é interpretado como índice de maturidade ou de precocidade e, também, é indicativo da velocidade de crescimento do animal; e 1/k está associado ao tempo necessário para atingir o peso de animal adulto, assim, quanto maior o valor do parâmetro $\mathrm{k}$ maior a precocidade do animal.

Em análises de medidas repetidas no tempo, como o peso a diferentes idades, pode haver autocorrelação positiva entre erros associados a idades próximas, além de heterogeneidade de variâncias dos pesos em razão da idade, o que pode levar a estimativas viesadas e com variâncias subestimadas (Pasternak \& Shalev, 1994; Souza, 1998). No ajuste do modelo Brody, considerouse a estrutura de erros autorregressivos de primeira ordem e a ponderação pelo inverso da variância residual de cinco classes de idade (Mazzini et al., 2005; Mariguele et al., 2011; Nascimento et al., 2011). Para a estimação dos parâmetros, utilizou-se o método dos mínimos quadrados e o algoritmo de Gauss-Newton. Para estas análises, utilizou-se o proc model, opção weight e macro \%AR, do programa SAS, versão 9.2 (SAS Institute, Cary, NC, EUA).

Aplicou-se o teste da razão de verossimilhança, para comparar o modelo completo ao reduzido, pois, com o modelo completo, é possível o ajuste de uma curva de crescimento específica para cada região de produção. Com o modelo reduzido, é possível ajustar uma única curva de crescimento, para todas as regiões de produção, ou curvas com subconjuntos de parâmetros comuns entre algumas regiões. Utilizou-se a metodologia, apresentada por Regazzi \& Silva (2010), para o teste de identidade de modelos de regressão não linear e de igualdade de qualquer subconjunto de parâmetros, por meio do teste da razão de verossimilhança, com aproximação pela estatística qui-quadrado, a 5\% de significância.

Para a realização do teste de razão da verossimilhança, criou-se uma variável indicadora (dummy) para a representação dos modelos, que assume valores binários 0 ou 1 ; assim, o modelo completo, com parâmetros diferentes para as cinco regiões de produção, é representado por

$$
\mathrm{y}_{\mathrm{ij}}=\sum_{\mathrm{j}=1}^{5} \mathrm{D}_{\mathrm{j}}\left[\mathrm{A}_{\mathrm{j}}\left(1-\mathrm{b}_{\mathrm{j}} \mathrm{e}^{-\mathrm{k}_{\mathrm{j}} \mathrm{x}_{\mathrm{ij}}}\right)+\varepsilon_{\mathrm{ij}}\right]
$$

em que: $D_{j}=1$, se o animal pertence à região de produção j; e $\mathrm{D}_{\mathrm{j}}=0$, se o animal não pertence à região de produção $\mathrm{j}$.

O modelo reduzido, com hipótese de igualdade dos parâmetros para todas as regiões de produção, que representa o ajuste de uma única curva de crescimento, é dado por

$$
\mathrm{y}_{\mathrm{ij}}=\sum_{\mathrm{j}=1}^{5} \mathrm{D}_{\mathrm{j}}\left[\mathrm{A}\left(1-\mathrm{be} \mathrm{ex}_{\mathrm{ij}}\right)+\varepsilon_{\mathrm{ij}}\right]=\mathrm{A}\left(1-\mathrm{be} \mathrm{ex}^{-k \mathrm{x}_{\mathrm{ij}}}\right)+\varepsilon_{\mathrm{ij}}
$$

Foram comparados, também, modelos reduzidos com hipótese de igualdade de subconjuntos de parâmetros. Por exemplo, apenas quanto ao parâmetro A, comum às cinco regiões de produção, o modelo ajustado é representado por:

$$
\mathrm{y}_{\mathrm{ij}}=\sum_{\mathrm{j}=1}^{5} \mathrm{D}_{\mathrm{j}}\left[\mathrm{A}\left(1-\mathrm{b}_{\mathrm{j}} \mathrm{e}^{-\mathrm{k}_{\mathrm{j}} \mathrm{x}_{\mathrm{ij}}}\right)+\varepsilon_{\mathrm{ij}}\right]
$$

Após o ajuste dos modelos, a estatística qui-quadrado $\left(\chi^{2}\right)$, para o teste da razão de verossimilhança, foi computada a partir dos valores máximos da função de verossimilhança para o modelo reduzido $(\mathrm{L} \omega)$ e o completo $(\mathrm{L} \Omega)$, dada por

$$
\chi^{2}=-2 \ln [\mathrm{L} \omega / \mathrm{L} \Omega]=[-2 \ln \mathrm{L} \Omega]
$$

com graus de liberdade, obtidos pela diferença entre o número de parâmetros dos modelos completo e reduzido.

\section{Resultados e Discussão}

O teste da razão de verossimilhança, quanto aos machos, foi significativo para o modelo reduzido com apenas quatro parâmetros, com hipótese de igualdade dos parâmetros (A, b e k) nas cinco regiões de produção (Tabela 2). Portanto, a diferença significativa entre este modelo reduzido e o completo com 16 parâmetros é indicativa de que o ajuste de uma única curva não é 
adequado, para a descrição do crescimento de machos, em todas as regiões de produção.

Os modelos reduzidos com 12 parâmetros, com hipótese de igualdade de apenas um dos três parâmetros (A, b ou k), nas cinco regiões de produção, também apresentaram diferença significativa e diferiram do modelo completo e, portanto, não foram adequados para descrever o crescimento de machos.

O teste da razão de verossimilhança não foi significativo quanto ao modelo reduzido com 14 parâmetros, com hipótese de taxa de maturidade (k) comum a grupos de regiões de produção. Portanto, este modelo não diferiu do completo e foi o mais adequado, pois apresentou dois parâmetros a menos em relação ao modelo completo. E de acordo com o princípio da parcimônia, modelos mais simples devem ser escolhidos em relação aos mais complexos.

De acordo com o modelo reduzido com 14 parâmetros, as regiões de produção com a mesma taxa de maturidade $(\mathrm{k})$ para machos foram: Gado Algodão e Mata Agreste $(\mathrm{k}=0,0009)$; e Maranhão e Itapetinga-Valadares $(\mathrm{k}=0,0016)$. Para a região Sertão, a taxa de maturidade estimada foi superior à das demais regiões $(\mathrm{k}=0,0027)$ (Tabela 3$)$.

As regiões de produção Gado Algodão e Mata Agreste apresentaram as maiores estimativas de peso assintótico (A) de machos, respectivamente, 847,75 e $846,04 \mathrm{~kg}$. Estas duas regiões também apresentaram a menor estimativa quanto à taxa de maturidade

Tabela 2. Teste da razão de verossimilhança, com aproximação de qui-quadrado $\left(\chi^{2}\right)$, para avaliar a identidade de modelos entre regiões de produção do Nordeste brasileiro, considerando-se o ajuste do modelo Brody para machos da raça Tabapuã ${ }^{(1)}$.

\begin{tabular}{|c|c|c|c|c|}
\hline Parâmetros $^{(2)}$ & $\mathrm{p}$ & $-2 \ln L$ & $\chi^{2}$ & Valor-P \\
\hline & \multicolumn{4}{|c|}{ Modelo completo } \\
\hline \multirow[t]{2}{*}{$\mathrm{A}_{\mathrm{j}} \mathrm{b}_{\mathrm{j}} \mathrm{k}_{\mathrm{j}} \varphi_{1}$} & 16 & $50.193,52$ & - & - \\
\hline & \multicolumn{4}{|c|}{ Modelos reduzidos (MR) } \\
\hline $\mathrm{Abk} \varphi_{1}$ & 4 & $50.533,46$ & $339,95 * *$ & $<0,0001$ \\
\hline$A b_{j} k_{j} \varphi_{1}$ & 12 & $50.366,46$ & $173,20 * *$ & $<0,0001$ \\
\hline $\mathrm{A}_{\mathrm{j}} \mathrm{b} \mathrm{k}_{\mathrm{j}} \varphi_{1}$ & 12 & $50.311,98$ & $118,46^{* *}$ & $<0,0001$ \\
\hline $\mathrm{A}_{\mathrm{j}} \mathrm{b}_{\mathrm{j}} \mathrm{k} \varphi_{1}$ & 12 & $50.307,16$ & $113,70 * *$ & $<0,0001$ \\
\hline \multicolumn{5}{|c|}{ MR com parâmetro k comum aos grupos: GA-AG; MA-IT e SE } \\
\hline $\mathrm{A}_{\mathrm{j}} \mathrm{b}_{\mathrm{j}} \mathrm{k}_{\mathrm{g}} \varphi_{1}$ & 14 & $50.198,06$ & $4,54^{\mathrm{ns}}$ & 0,10 \\
\hline
\end{tabular}

(1)MA, Maranhão; GA, Gado Algodão; AG, Mata Agreste; SE, Sertão; IT, Itapetinga-Valadares. ${ }^{(2)}$ Parâmetros do modelo Brody, com autocorrelação residual de primeira ordem $\left(\varphi_{1}\right)$. p, número de parâmetros nos modelos completo e reduzido, com pelo menos um parâmetro comum entre regiões. $(\mathrm{k}=0,0009)$, o que é indicativo de maior tempo para os animais atingirem o peso adulto, enquanto na região de produção Sertão os animais atingiram o menor peso adulto $(370,27 \mathrm{~kg})$, porém precocemente. Estes resultados também são indicativos de correlação negativa entre os parâmetros peso adulto (A) e taxa de maturidade $(\mathrm{k})$.

De acordo com o teste da razão de verossimilhança, os modelos reduzidos com 4 e 12 parâmetros diferiram significativamente do modelo completo para as fêmeas (Tabela 4). Todavia, o modelo reduzido com 14 parâmetros, com hipótese de taxa de maturidade comum a grupos de regiões de produção, não diferiu do completo. Portanto, para ambos os sexos, o modelo com o mesmo valor do parâmetro taxa de maturidade,

Tabela 3. Estimativas e erros-padrão aproximados, para parâmetros do modelo reduzido com taxa de maturidade $(\mathrm{k})$ comum aos grupos de regiões de produção (RP) GA-AG; MA-IT e SE, considerando-se o ajuste do modelo Brody para machos da raça Tabapuã.

\begin{tabular}{lcccc}
\hline $\mathrm{RP}^{(1)}$ & $\mathrm{A}$ & $\mathrm{b}$ & $\mathrm{k}$ & $\hat{\varphi}_{1}{ }^{(2)}$ \\
\hline GA & $847,75(40,67)$ & $0,95(0,0032)$ & \multirow{2}{*}{$0,0009(0,00006)$} & \\
AG & $846,04(39,74)$ & $0,96(0,0018)$ & & \\
\hline MA & $575,21(14,53)$ & $0,95(0,0089)$ & \multirow{2}{*}{$0,0016(0,00003)$} & $(0,0040)$ \\
IT & $540,10(05,66)$ & $0,94(0,0008)$ & & \\
\hline SE & $370,27(21,65)$ & $0,93(0,0129)$ & $0,0027(0,0003)$ & \\
\hline
\end{tabular}

(1)MA, Maranhão; GA, Gado Algodão; AG, Mata Agreste; SE, Sertão; IT, Itapetinga-Valadares. (2) $\hat{\varphi}_{1}$, parâmetro de autocorrelação residual de primeira ordem.

Tabela 4. Teste da razão de verossimilhança, com aproximação de qui-quadrado $\left(\chi^{2}\right)$, para avaliar a identidade de modelos entre regiões de produção ${ }^{(1)}$ do Nordeste brasileiro, considerando-se o ajuste do modelo Brody para fêmeas da raça Tabapuã.

\begin{tabular}{lcccc}
\hline Parâmetro $^{(2)}$ & $p$ & $-2 \operatorname{lnL}$ & $\chi^{2}$ & Valor-P \\
\hline \multicolumn{5}{c}{ Modelo completo } \\
$\mathrm{A}_{\mathrm{j}} \mathrm{b}_{\mathrm{j}} \mathrm{k}_{\mathrm{j}} \varphi_{1}$ & 16 & $57.812,80$ & - & - \\
\hline \multicolumn{5}{c}{ Modelos reduzidos (MR) } \\
$\mathrm{A} \mathrm{b} \mathrm{k} \varphi_{1}$ & 4 & $58.027,86$ & $215,06^{* *}$ & $<0,0001$ \\
$\mathrm{~A} \mathrm{~b}_{\mathrm{j}} \mathrm{k}_{\mathrm{j}} \varphi_{1}$ & 12 & $57.912,70$ & $99,90^{* *}$ & $<0,0001$ \\
$\mathrm{~A}_{\mathrm{j}} \mathrm{b} \mathrm{k} \mathrm{k}_{\mathrm{j}} \varphi_{1}$ & 12 & $57.822,90$ & $10,10^{*}$ & 0,0388 \\
$\mathrm{~A}_{\mathrm{j}} \mathrm{b}_{\mathrm{j}} \mathrm{k} \varphi_{1}$ & 12 & $57.880,70$ & $67,90^{* *}$ & $<0,0001$ \\
\hline \multicolumn{5}{c}{ MR com parâmetro k comum aos grupos: AG-SE; MA-IT e GA } \\
$\mathrm{A}_{\mathrm{j}} \mathrm{b}_{\mathrm{j}} \mathrm{k}_{\mathrm{g}} \varphi_{1}$ & 14 & $57.813,88$ & $1,08^{\mathrm{ns}}$ & 0,5827 \\
\hline
\end{tabular}

(1)MA, Maranhão; GA, Gado Algodão; AG, Mata Agreste; SE, Sertão; IT, Itapetinga-Valadares. ${ }^{(2)}$ Parâmetros do modelo Brody, com autocorrelação residual de primeira ordem $\left(\varphi_{1}\right)$. p, número de parâmetros nos modelos completo e reduzido, com pelo menos um parâmetro comum entre regiões. 
para regiões de produção, foi o mais adequado para descrever o crescimento dos animais. Porém, a formação dos grupos de regiões foi diferente para machos e fêmeas.

Quanto às fêmeas, as regiões com a mesma taxa de maturidade foram Mata Agreste e Sertão, Maranhão e Itapetinga-Valadares, e Gado Algodão (Tabela 5). As fêmeas apresentaram os menores pesos assintóticos estimados (376,97 a 478,03 kg) e as maiores estimativas para taxa de maturidade $(0,0017$ a 0,0027$)$, como se esperava, em razão da maior precocidade e do menor peso adulto de fêmeas em comparação a machos.

As regiões de produção com maiores estimativas de peso assintótico apresentaram as menores estimativas para taxa de maturidade (Tabelas 3 e 5), o que indica correlação negativa entre estes parâmetros, aspecto bastante destacado por outros autores (Freitas, 2005; Garnero et al., 2005; Santoro et al., 2005; Souza et al., 2010; Ibáñez-Escriche \& Blasco, 2011). As estimativas de peso assintótico e taxa de maturidade variaram muito entre as cinco regiões de produção, o que é uma indicação de que uma única curva pode não ser adequada para descrever o crescimento dos animais, nas cinco regiões de produção do Nordeste.

As estimativas obtidas para os parâmetros da curva de crescimento, para ambos os sexos da raça Tabapuã (Tabelas 3 e 5), apresentaram maior amplitude do que as relatadas por outros autores sobre animais de outras raças zebuínas, tais como Guzerá, Nelore e Indubrasil (Santoro et al., 2005; Souza et al., 2010; Silva, et al., 2011a, 2011b; Garnero et al., 2013). Estes autores também obtiveram, com ajuste do modelo Brody, o peso de animal adulto estimado próximo de $500 \mathrm{~kg}$, com taxa de maturidade de 0,0015 a 0,0024. Ressalta-

Tabela 5. Estimativas e erros-padrão aproximados, para parâmetros do modelo reduzido com taxa de maturidade $(\mathrm{k})$ comum aos grupos de regiões de produção (RP): AG-SE; MA-IT e GA, considerando-se o ajuste do modelo Brody para fêmeas da raça Tabapuã.

\begin{tabular}{|c|c|c|c|c|}
\hline $\mathrm{RP}^{(1)}$ & $\mathrm{A}$ & $\mathrm{b}$ & $\mathrm{k}$ & $\hat{\varphi}_{1}^{(2)}$ \\
\hline$\overline{\mathrm{AG}}$ & $478,03(09,62)$ & $0,93(0,0020)$ & \multirow{2}{*}{$0,0017(0,00006)$} & \multirow{5}{*}{$0,60(0,0035)$} \\
\hline SE & $408,24(11,21)$ & $0,92(0,0088)$ & & \\
\hline$\overline{\mathrm{MA}}$ & $416,33(04,47)$ & $0,92(0,0041)$ & \multirow{2}{*}{$0,0022(0,00003)$} & \\
\hline IT & $394,29(02,58)$ & $0,93(0,0010)$ & & \\
\hline$\overline{\mathrm{GA}}$ & $376,97(12,28)$ & $0,93(0,0065)$ & $0,0027(0,00016)$ & \\
\hline
\end{tabular}

(1)MA, Maranhão; GA, Gado Algodão; AG, Mata Agreste; SE, Sertão; IT, Itapetinga-Valadares. ${ }^{(2)} \varphi_{1}$, parâmetro de autocorrelação residual de primeira ordem. se que estas estimativas foram obtidas com o ajuste de uma única curva, para animais criados em vários estados ou em um único estado, como nos trabalhos de Santoro et al. (2005) e Souza et al. (2010) sobre animais criados nos estados de Pernambuco e Sergipe, respectivamente.

O Estado de Pernambuco contém três regiões de produção (Gado Algodão, Mata Agreste e Sertão). Para estas regiões, as estimativas de peso assintótico e taxa de maturidade para machos variaram de 370,27 a $847,75 \mathrm{~kg}$, e de 0,0009 a 0,0027 , respectivamente (Tabela 3). Com estes resultados, confirmou-se que até no mesmo estado de criação, o ajuste de uma única curva de crescimento não é adequado para descrever o crescimento dos animais.

Silva et al. (2011a) ajustaram curvas de crescimento, com uso do modelo Brody, para fêmeas da raça zebuína Nelore, criadas na região Sudeste. Os autores observaram que as estimativas quanto a peso assintótico e taxa de maturidade foram respectivamente, $493,27 \mathrm{~kg}$ e 0,0018. Resultados semelhantes foram obtidos por Oliveira et al. (2000), Santoro et al. (2005), Souza et al. (2010) e Silva et al. (2011b), no estudo de crescimento de fêmeas das raças Guzerá, Nelore e Indubrasil. No presente estudo, as estimativas para fêmeas da raça Tabapuã, quanto ao peso assintótico, foram inferiores às estimativas obtidas por esses autores, enquanto as estimativas para taxa de maturidade foram, em geral, superiores (Tabela 5).

\section{Conclusões}

1. A utilização de uma única curva para descrever o crescimento de bovinos da raça Tabapuã, nas cinco regiões de produção do Nordeste brasileiro, não é adequada.

2. Para machos, as curvas de crescimento apresentam taxas de maturidade em comum nas regiões de produção Gado Algodão e Mata Agreste, Maranhão e Itapetinga-Valadares, e Sertão.

3. Para fêmeas, as curvas de crescimento apresentam taxas de maturidade em comum nas regiões de produção Mata Agreste e Sertão, Maranhão e Itapetinga-Valadares, e Gado Algodão.

\section{Agradecimentos}

Ao Conselho Nacional de Desenvolvimento Científico e Tecnológico (CNPq), por concessão de 
bolsas; ao Programa de Pós-graduação em Estatística e Experimentação Agropecuária, da Universidade Federal de Lavras; à Associação Brasileira de Criadores de Zebu (ABCZ); e à Fundação de Amparo à Pesquisa do Estado da Bahia (Fapesb), pelo apoio financeiro.

\section{Referências}

ARRUDA, Z.J. de; SUGAI, Y. Regionalização da pecuária bovina no Brasil. Campo Grande: Embrapa-CNPGC; Brasília: Embrapa-SPI, 1994. 144p.

FORNI, S.; PILES, M.; BLASCO, A.; VARONA, L.; OLIVEIRA, H.N.; LÔBO, R.B.; ALBUQUERQUE, L.G. Analysis of beef cattle longitudinal data applying a nonlinear model. Journal of Animal Science, v.85, p.3189-3197, 2007. DOI: 10.2527/jas.2006-677.

FREITAS, A.R. de. Curvas de crescimento na produção animal. Revista Brasileira de Zootecnia, v.34, p.786-795, 2005. DOI: 10.1590/S1516-35982005000300010.

GARNERO, A. del V.; MARCONDES, C.R.; ARAÚJO, R.O. de; OLIVEIRA, H.N. de; LÔBO, R.B. Inferência bayesiana aplicada à estimação de herdabilidades dos parâmetros da curva de crescimento de fêmeas da raça Nelore. Ciência Rural, v.43, p.702-708, 2013. DOI: 10.1590/S0103-84782013005000029.

GARNERO, A. del V.; MARCONDES, C.R.; BEZERRA, L.A.F.; OLIVEIRA, H.N.; LÔBO, R.B. Parâmetros genéticos da taxa de maturação e do peso assintótico de fềmeas da raça Nelore. Arquivo Brasileiro de Medicina Veterinária e Zootecnia, v.57, p.652-662, 2005. DOI: 10.1590/S0102-09352005000500011.

IBÁÑEZ-ESCRICHE, N.; BLASCO, A. Modifying growth curve parameters by multitrait genomic selection. Journal of Animal Science, v.89, p.661-668, 2011. DOI: 10.2527/jas.2010-2984.

MALHADO, C.H.M.; RAMOS, A.A.; CARNEIRO, P.L.S.; AZEVEDO, D.M.M.R.; MELLO, P.R.A. de; PEREIRA, D.G.; SOUZA, J.C. de; MARTINS FILHO, R. Modelos não lineares utilizados para descrever o crescimento de bovinos da raça Nelore no Estado da Bahia: 1. Efeito ambiental. Revista Brasileira de Saúde e Produção Animal, v.10, p.821-829, 2009.

MARIGUELE, K.H.; RESENDE, M.D.V. de; VIANA, J.M.S.; SILVA, F.F. e; SILVA, P.S.L. de; KNOP, F. de C. Métodos de análise de dados longitudinais para o melhoramento genético da pinha. Pesquisa Agropecuária Brasileira, v.46, p.1657-1664, 2011. DOI: 10.1590/S0100-204X2011001200011.

MARTINS, F.B.; SOARES, C.P.B.; LEITE, H.S.; SOUZA, A.L. de; CASTRO, R.V.O. Índices de competição em árvores individuais de eucalipto. Pesquisa Agropecuária Brasileira, v.46, p.1089-1098, 2011. DOI: 10.1590/S0100-204X2011000900017.

MAZZINI, A.R. de A.; MUNIZ, J.A.; SILVA, F.F. e; AQUINO, L.H. de. Curva de crescimento de novilhos Hereford: heterocedasticidade e resíduos autorregressivos. Ciência Rural, v.35, p.422-427, 2005. DOI: 10.1590/S0103-84782005000200028.
NASCIMENTO, M.; SÁFADI, T.; SILVA, F.F. e. Aplicação da análise de agrupamento de dados de expressão gênica temporal a dados em painel. Pesquisa Agropecuária Brasileira, v.46, p.1489-1495, 2011. DOI: 10.1590/ S0100-204X2011001100010.

OLIVEIRA, H.N. de; LÔBO, R.B.; PEREIRA, C.S. Comparação de modelos não-lineares para descrever o crescimento de fêmeas da raça Guzerá. Pesquisa Agropecuária Brasileira, v.35, p.1843-1851, 2000. DOI: 10.1590/S0100-204X2000000900017.

PASTERNAK, H.; SHALEV, B.A. The effect of a feature of regression disturbance on the efficiency of fitting growth curves. Growth, Development and Aging, v.58, p.33-39, 1994.

RATKOWSKY, D.A. Handbook of nonlinear regression models. New York: Marcel Dekker, 1990. 241p.

REGAZZI, A.J; SILVA, C.H.O. Testes para verificar a igualdade de parâmetros e a identidade de modelos de regressão não-linear em dados de experimento com delineamento em blocos casualizados. Revista Ceres, v.57, p.315-320, 2010. DOI: 10.1590/ S0034-737X2010000300005.

SANTORO, K.R.; BARBOSA, S.B.P.; BRASIL, L.H. de A.; SANTOS, E. de S. Estimativas de parâmetros de curvas de crescimento de bovinos Zebu, criados no Estado de Pernambuco. Revista Brasileira de Zootecnia, v.34, p.2262-2279, 2005. DOI: 10.1590/S1516-35982005000700013.

SANTOS, T.C.; MURAKAMI, A.E.; OLIVEIRA, C.A.L.; COSTA, P.D. Desenvolvimento corporal e testicular em machos de codornas de corte e de postura de 25 a 360 dias. Pesquisa Veterinária Brasileira, v.32, p.1205-1212, 2012. DOI: 10.1590/ S0100-736X2012001100023.

SARMENTO, J.L.R.; REZAZZI, A.J.; SOUZA, W.H. de; TORRES, R. de A.; BREDA, F.C.; MENEZES, G.R. de O. Estudo da curva de crescimento de ovinos Santa Inês. Revista Brasileira de Zootecnia, v.35, p.435-442, 2006. DOI: 10.1590/ S1516-35982006000200014.

SILVA, F. de L.; ALENCAR, M.M. de; FREITAS, A.R. de; PACKER, I.U.; MOURÃO, G.B. Curvas de crescimento em vacas de corte de diferentes tipos biológicos. Pesquisa Agropecuária Brasileira, v.46, p.262-271, 2011a. DOI: 10.1590/S0100204X2011000300006.

SILVA, N.A.M.; LANA, A.M.Q.; SILVA, F.F.; SILVEIRA, F.G.; BERGMANN, J.A.G.; SILVA, M.A.; TORAL, F.L.B. Seleção e classificação multivariada de modelos de crescimento não lineares para bovinos Nelore. Arquivo Brasileiro de Medicina Veterinária e Zootecnia, v.63, p.364-371, 2011b. DOI: 10.1590/ S0102-09352011000200014.

SOUZA, G. da S. e. Introdução aos modelos de regressão linear e não-linear. Brasília: Embrapa SPI: Embrapa-seA, 1998. 489p.

SOUZA, L. de A.; CAIRES, D.N.; CARNEIRO, P.L.S.; MALHADO, C.H.M.; MARTINS FILHO, R. Curvas de crescimento em bovinos da raça Indubrasil criados no Estado de Sergipe. Revista Ciência Agronômica, v.41, p.671-676, 2010. DOI: $10.1590 /$ S1806-66902010000400022.

Recebido em 19 de agosto de 2013 e aprovado em 23 de dezembro de 2013

Pesq. agropec. bras., Brasília, v.49, n.1, p.57-62, jan. 2014

DOI: $10.1590 / \mathrm{S} 0100-204 X 2014000100008$ 